\title{
Electronic structure and optical properties of quantum crystals from first principles calculations in the Born-Oppenheimer approximation
}

\author{
Vitaly Gorelov, ${ }^{1}$ David M. Ceperley, ${ }^{2}$ Markus Holzmann, ${ }^{3,4}$ and Carlo Pierleoni ${ }^{1,5}$ \\ 1) Maison de la Simulation, CEA, CNRS, Univ. Paris-Sud, UVSQ, Université Paris-Saclay, 91191 Gif-sur-Yvette, \\ France \\ ${ }^{2)}$ Department of Physics, University of Illinois, Urbana, Illinois 61801, USA \\ 3) Univ. Grenoble Alpes, CNRS, LPMMC, 3800 Grenoble, France \\ ${ }^{4)}$ Institut Laue Langevin, BP 156, F-38042 Grenoble Cedex 9, France \\ ${ }^{5)}$ Department of Physical and Chemical Sciences, University of L'Aquila, Via Vetoio 10, I-67010 L'Aquila, \\ Italy
}

(Dated: 6 October 2020)

We develop a formalism to accurately account for the renormalization of electronic structure due to quantum and thermal nuclear motions within the Born-Oppenheimer approximation. We focus on the fundamental energy gap obtained from electronic addition and removal energies from Quantum Monte Carlo calculations in either the canonical or grand canonical ensembles. The formalism applies as well to effective single electron theories such as those based on Density Functional Theory. We show that electronic (Bloch) crystal momentum can be restored by marginalizing the total electron-ion wave function with respect to the nuclear equilibrium distribution, and we describe an explicit procedure to establish the band structure of electronic excitations for quantum crystals within the Born-Oppenheimer approximation. Based on the Kubo-Greenwood equation, we discuss the effects of nuclear motion on optical conductivity. Our methodology applies to the low temperature regime where nuclear motion is quantized and in general differs from the semi-classical approximation. We apply our method to study the electronic structure of C2/c-24 crystalline hydrogen at $200 \mathrm{~K}$ and $250 \mathrm{GPa}$ and discuss the optical absorption profile of hydrogen crystal at $200 \mathrm{~K}$ and carbon diamond at $297 \mathrm{~K}$.

\section{INTRODUCTION}

With increasing computational power, precise Quantum Monte Carlo (QMC) calculations of electronic properties in real materials have become affordable ${ }^{1-9}$. Quantum Monte Carlo methods naturally extend to solve the full Schrödinger equation of the coupled electron-ion system at zero temperature ${ }^{10-12}$ or at finite temperature within the Path Integral formalism ${ }^{13-15}$. For typical temperatures in condensed matter, the Born-Oppenheimer approximation can be further used to sample the nuclear distribution either within coupled electron ion Monte Carlo (CEIMC) ${ }^{16}$ or molecular dynamics simulations ${ }^{17,18}$.

Recently, we studied the effect of nuclear quantum and thermal motion on the closure of the fundamental electronic gap in high-pressure solid and liquid hydrogen within CEIMC $^{19,20}$. Here, we discuss how the fundamental gap and the band structure can be obtained for quantum and thermal crystals in a fully non-perturbative approach using QMC based methods. We further propose a new scheme to effectively include nuclear quantum and thermal effects on optical properties at low temperature where the usual semiclassical approximation breaks down.

Standard electronic structure methods based on effective single electron theories like Density Functional Theory (DFT), or many-body perturbation theory (GW), often assume a weak electron-phonon coupling and phonons within the harmonic approximation so that their effects on the electronic structure can be treated perturbatively ${ }^{21-33}$, based on the seminal work of Allen, Heine, and Cordona ${ }^{34-36}$. Both assumptions ${ }^{37}$ limit the predictive power of these methods, especially for systems of light nuclei, like solid hydrogen ${ }^{38,39}$ and other molecular crystals ${ }^{40}$. To go beyond the harmonic limit, one can employ the self-consistent harmonic approximation (SCHA), an effective mean field theory based on minimizing a free energy bound with respect to the effective equilibrium nuclear positions and effective frequencies within an harmonic ansatz for the nuclear motion. One characteristic of SCHA is to allow for structural predictions induced by nuclear quantum and thermal effects ${ }^{41,42}$, but is intrinsically difficult to improve on further.

Non-perturbative treatment of phonons can be achieved by path-integral calculations of the nuclear motion within the Born-Oppenheimer approximation. Path integral molecular dynamics (PIMD) and Monte Carlo (PIMC) has been used to study the renormalization of electronic structure of different materials due to nuclear motion mainly based on a semiclassical interpretation of the instantaneous Born-Oppenheimer electronic energies of the nuclear trajectory ${ }^{43-50}$. In this scheme, nuclear coordinates have been implicitly assumed to be good quantum numbers for the nuclear motion. However, this is questionable at low temperatures where quantization of phonons is important. Here, we will show how to take properly into account the renormalization of electronic structure at low temperatures within PIMD and PIMC extending the discussion anticipated in Ref. ${ }^{19}$.

Concerning optical properties, ab-initio calculations based on the Born-Oppenheimer approximation typically employ the semi-classical William-Lax approach ${ }^{51,52}$, where the optical spectra computed at fixed nuclear configuration are averaged over the equilibrium nuclear distribution. Successfully applied to study optical properties 
of heavier elements ${ }^{53,54}$, its accuracy for light elements like hydrogen remains questionable and we will discuss the minimal changes needed at low temperatures when nuclear motion is quantized.

The paper is organized as follows. In section II we discuss the energy gaps and band structure of quantum crystals in a many-body framework. We first define the fundamental gap based on electron addition and removal energies within the $\mathrm{BO}$ approximation in the canonical and grand canonical ensemble. We then show how the concept of crystal momentum of electronic excitations can be meaningfully extended to the case of quantum crystals in a fully non-perturbative way, and finally present some illustrative results for solid hydrogen at $200 \mathrm{~K}$ and 250 GPa. In section III we then study the effects of finite temperature and zero point nuclear motion on the optical properties for semiclassical and quantum nuclei and compute optical absorption of solid hydrogen and carbon diamond at finite temperature. In section IV we summarize our results. Explicit steps involved in the calculations of the density of states (DOS) in the grand canonical ensemble are detailed in the Appendix.

\section{ENERGY GAP AND BAND STRUCTURE}

In the following we define and discuss single particle electronic excitation energies focusing on the fundamental electronic energy gap and the band structure in a quantum crystal at zero and finite temperatures. We will assume the validity of the Born-Oppenheimer approximation, a simplification usually well justified in the description of condensed-matter. We stress that the formalism equally applies to many-body and effective single-electron theories.

\section{A. Canonical ensemble}

Let us consider a system with $N_{p}$ protons and $N_{e}$ electrons, mutually interacting via the bare Coulomb interaction. To assure global charge neutrality, a uniform charge background is added whenever $N_{p} \neq N_{e}$. The canonical partition function at nuclear temperature $T=$ $1 / k_{B} \beta$ and volume $V$ can be written as a path-integral

$$
Z\left(N_{e}\right)=e^{-\beta F\left(N_{e}\right)}=\int \mathcal{D} \mathbf{R}(\tau) e^{-S[\mathbf{R}(\tau)]}
$$

where the action is

$$
S[\mathbf{R}(\tau)]=\int_{0}^{\beta} d \tau\left[\frac{\hbar^{2}}{2 M}\left(\frac{d \mathbf{R}(\tau)}{d \tau}\right)^{2}+E_{0}\left(\mathbf{R}(\tau), N_{e}\right)\right] .
$$

Here $M$ is the nuclear mass, $\mathbf{R}$ the vector of the $N_{p}$ nuclear coordinates, and $E_{0}\left(\mathbf{R}, N_{e}\right)$ denotes the electronic BornOppenheimer potential energy surface (PES) calculated with $N_{e}$ electrons. As we will concentrate on situations

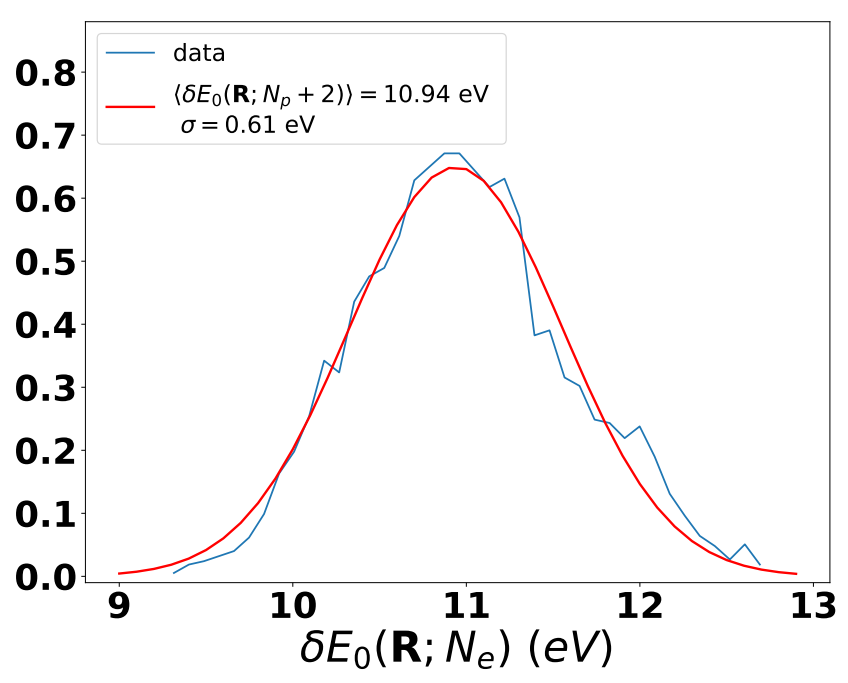

FIG. 1: Histogram of the distribution of energy needed to add two electrons to $\mathrm{C} 2 / \mathrm{c}-24$ solid hydrogen at $200 \mathrm{~K}$ and $248 \mathrm{GPa}$ compared with a normal distribution.

where we only vary the number of electrons $N_{e}$ keeping $V, T$, and $N_{P}$ constant, we do not explicitly write out the dependence on these latter variables.

Within CEIMC calculations the imaginary time discretized path-integral is typically calculated for the undoped situation, $N_{e}=N_{p}$, and the free energy difference can be obtained from

$$
\frac{Z\left(N_{e}\right)}{Z\left(N_{p}\right)}=e^{-\beta\left[F\left(N_{e}\right)-F\left(N_{p}\right)\right]}=\left\langle e^{-\int_{0}^{\beta} d \tau \delta E_{0}\left(\mathbf{R}(\tau), N_{e}\right)}\right\rangle
$$

where $\delta E_{0}\left(\mathbf{R}, N_{e}\right)=E_{0}\left(\mathbf{R}, N_{e}\right)-E_{0}\left(\mathbf{R}, N_{p}\right)$ and $\langle\cdots\rangle$ denotes the averaging over the undoped sample, $N_{e}=N_{p}$.

For small doping, $\left|N_{e}-N_{p}\right| \ll N_{p}$, the potential energy surface should only be slightly perturbed, $\left|\delta E_{0}\left(\mathbf{R}, N_{e}\right)\right| \ll$ $\left|E_{0}\left(\mathbf{R}, N_{p}\right)\right|$, and we may use the cumulant expansion (see Fig. 1), which gives

$$
\begin{gathered}
F\left(N_{e}\right)-F\left(N_{p}\right)=\left\langle\delta E_{0}\left(\mathbf{R}(0), N_{e}\right)\right\rangle-\frac{\sigma^{2}\left(N_{e}\right)}{2} \\
\sigma^{2}\left(N_{e}\right)=\int_{0}^{\beta} d \tau\left\langle\delta E_{0}\left(\mathbf{R}(\tau), N_{e}\right) \delta E_{0}\left(\mathbf{R}(0), N_{e}\right)\right\rangle_{c}
\end{gathered}
$$

where $\langle\ldots\rangle_{c}$ indicates the centered cumulant. Having the form of a static response function, $\sigma^{2}\left(N_{e}\right)$ describes the leading order changes of the adding/removal energies due to the electronically doped potential energy surface.

Adding (or removing) a single electron, we obtain the chemical potential from the free energy differences

$$
\mu_{ \pm}=F\left(N_{p} \pm 1\right)-F\left(N_{p}\right) .
$$

A gap with respect to electronic doping, $\Delta=\mu_{+}-\mu_{-}$, can be determined from thermodynamics.

Time-correlations of electron addition/removal energies described by $\sigma^{2}\left(N_{p} \pm 1\right)$ contribute with opposite sign to 
the gap and are expected to largely cancel each other,

$$
\Delta \simeq\left\langle\delta E_{0}\left(N_{p}+1\right)\right\rangle-\left\langle\delta E_{0}\left(N_{p}-1\right)\right\rangle
$$

so that the gap is entirely determined by the electronic addition and removal energies, as intuitively expected.

\section{B. Grand canonical ensemble}

In the grand canonical ensemble, instead of the electronic density, the (electronic) chemical potential $\mu$ is the independent variable. Since the integrated density of states is given by the density as a function $\mu$, a nonvanishing gap implies a vanishing of the density of states in the region $\mu_{-}<\mu<\mu_{+}$.

The use of twist averaged boundary conditions provides an efficient strategy to reduce the finite size effects that result from the discrete nature of filling single particle orbitals $^{55-59}$. In the following we describe its use in the grand canonical approach of calculating the fundamental $\operatorname{gap}^{7,19}$.

Expressed in terms of the canonical partition function $Z\left(N_{e}, \theta\right)$, the grand canonical partition function is

$$
\begin{aligned}
Z_{g c}(\mu, \theta) & =\sum_{N_{e}} e^{\beta \mu N_{e}} Z\left(N_{e}, \theta\right) \\
& =\sum_{N_{e}} e^{-\beta\left(F\left(N_{e}, \theta\right)-\mu N_{e}\right)}
\end{aligned}
$$

where we have explicitly added the dependence on the twist angle $\theta$ imposed on the boundary conditions of the electronic wave functions.

Consistent with the $\mathrm{BO}$ approximation, we address situations where the temperature is much less than the electronic excitation energy, so that in equilibrium one maximises the exponent in Eq. (8)

$$
\begin{aligned}
-T \log Z_{g c}(\mu, \theta) & =\min _{N_{e}}\left[F\left(N_{e}, \theta\right)-\mu N_{e}\right] \\
& \equiv F\left(N_{e}^{\theta}, \theta\right)-\mu N_{e}^{\theta}
\end{aligned}
$$

where $N_{e}^{\theta}$ denotes the number of electrons which minimizes the r.h.s. of Eq. (9). Although not explicitly indicated, let us stress that the finite temperature effects of the nuclear motion are still contained in the temperature dependence of $F\left(N_{e}^{\theta}, \theta\right)$.

Neglecting modifications of the potential energy surface due to doping, e.g. $\sigma^{2}$ in the free energy differences Eq. (4) as discussed previously, $N_{e}^{\theta}$ can be determined by replacing the free energy $F\left(N_{e}, \theta\right)$ with the electronic energy $E\left(N_{e}, \theta\right)$ in the minimization Eq. (9).

To reduce finite size effects, different independent calculations are performed over a dense grid of $M$ twist angles and the results are then averaged,

$$
\begin{aligned}
f(\mu) & =\frac{1}{M V} \sum_{\theta} F\left(N_{e}^{\theta}, \theta\right) \\
n_{e}(\mu) & =\frac{1}{M V} \sum_{\theta} N_{e}^{\theta} \\
e(\mu) & =\frac{1}{M V} \sum_{\theta} E_{0}\left(N_{e}^{\theta}, \theta\right)
\end{aligned}
$$

In the independent particle approximation, the free energy, the internal energy and electronic densities, $f, e$, and $n_{e}$ respectively, exactly agree with those obtained in a supercell of volume $M V$. In a many body theory, however, size effects due to correlations of two or more electrons must be addressed differently ${ }^{7,56,58}$.

A gap in the many-particle density of states for $\mu_{-}<$ $\mu<\mu_{+}$then implies a vanishing slope in fundamental thermodynamic properties $\left(f(\mu), n_{e}(\mu), e(\mu)\right)$ as a function of $\mu$ in this region. The value of the gap

$$
\Delta=\mu_{+}-\mu_{-}
$$

can be directly read off from the flat region in the plot of these functions.

We stress that the calculation of the thermodynamic potentials involves the average over nuclear configurations. This average should always be done before determining the fundamental gap. Hence the correct value of the fundamental gap is, in general, different (larger) than the result obtained by determining the gap from the minimum value of electronic excitation energies with respect to all nuclear configurations in a simulation run. The result of the latter procedure, which we call the "semiclassical approximation", may be justified when analysing spectroscopic quantities but does not necessarily represent the thermodynamic gap.

\section{Electronic band structure in quantum crystals}

Within effective single particle theories, the electronic structure of perfect crystals is conveniently described by discussing the resulting band structure of the electronic excitation energies as a function of their Bloch wave vector. Since the Bloch wave vector characterizes the symmetry properties of the underlying nuclear crystal, electronic addition and removal energies of a perfect crystal can still be labelled by its quasi-momentum also within a many-electron description ${ }^{60,61}$.

In the following we discuss the extension of the concept of quasi-momentum to the case of quantum crystals, where the nuclear positions fluctuate around their perfect crystal lattice sites, $\mathbf{R}_{0}$, with zero point quantum and/or thermal nuclear motion. 


\section{Harmonic approximation}

Let us first consider the case of small nuclear fluctuations around the lattice sites, such that the harmonic approximation can be used. Treating the electron-ion interaction as a weak perturbation, the $\alpha^{\text {th }}$ electronic wave function $\Phi_{\alpha}(\mathbf{r} \mid \mathbf{R})$ for a given (fixed) nuclear configuration $\mathbf{R}$ is given in first order in perturbation theory as

$$
\Phi_{a}(\mathbf{r} \mid \mathbf{R}) \simeq \Phi_{\mathbf{k} 0}^{\mathbf{R}_{0}}(\mathbf{r})+\sum_{\mathbf{q} m} \frac{\left(\mathbf{R}-\mathbf{R}_{0}\right) \cdot \mathbf{A}_{\mathbf{k} 0}^{\mathbf{q} m}}{E_{\mathbf{k} 0}^{\mathbf{R}_{0}}-E_{\mathbf{q} m}^{\mathbf{R}_{0}}} \Phi_{\mathbf{q} m}^{\mathbf{R}_{0}}(\mathbf{r})(15)
$$

Here, $\Phi_{\mathbf{k} n}^{\mathbf{R}_{0}}(\mathbf{r}) \equiv\left\langle\mathbf{r} \mid \Phi_{\mathbf{k} n}^{\mathbf{R}_{0}}\right\rangle$ denotes the electronic (ground state) wave function the perfect crystal, characterized by a (total) quasi-momentum $\mathbf{k}$ and a band index $n=0$. $\mathbf{r}$ represents all electronic coordinates. With nuclear displacements, $\Phi(\mathbf{r} \mid \mathbf{R})$ will have contributions from electronic excited states $(\mathbf{q} m) \neq(\mathbf{k} 0)$ of the periodic Hamiltonian, and $\mathbf{A}_{\mathbf{k} 0}^{\mathbf{q} m}=\nabla_{\mathbf{R}}\left\langle\Phi_{\mathbf{q} m}^{\mathbf{R}_{0}}|H| \Phi_{\mathbf{k} 0}^{\mathbf{R}_{0}}\right\rangle$ denotes the matrix elements evaluated at $\mathbf{R}=\mathbf{R}_{0}$. Only the electron-nuclear interaction in the Hamiltonian $H$ contribute to this matrix elements.

Integrating $\mathrm{Eq}$ (15) over the distribution nuclear fluctuations with $\left\langle R-R_{0}\right\rangle=0$, e.g. zero-point or thermal fluctuations, the contribution of electronic excited states drops out:

$$
\left\langle\Phi_{\alpha}(\mathbf{r} \mid \mathbf{R})\right\rangle=\phi_{\mathbf{k} 0}^{\mathbf{R}_{0}}(r) .
$$

The symmetry of the perfect crystal is thus restored, and, at least in the harmonic approximation, we can characterize the $\alpha^{t h}$ electronic wave function of the quantum crystal by the quasi-momentum of the adiabatically connected state $\mathbf{k}$,

$$
\left\langle\Phi_{\alpha}(\mathbf{r}+\mathbf{a} \mid \mathbf{R})\right\rangle=e^{i \mathbf{k} \cdot \mathbf{a}}\left\langle\Phi_{\alpha}(\mathbf{r} \mid \mathbf{R})\right\rangle
$$

where a denotes any of the crystal lattice vectors.

In general, the electronic ground state of the system will have a vanishing quasi-momentum in a large enough supercell, but adding and removal energies are still characterized by a quasi-momentum within the first Brillouin zone (BZ) even for a quantum crystal. It remains a useful concept for analysing transition matrix elements of purely electronic operators: as long as the nuclear distribution remains unchanged in the transition matrix, the quasimomentum must be conserved, and the usual selection rules for perfect crystals apply. In particular, one may distinguish between direct and indirect transition according to the Bloch vectors involved.

\section{Beyond the harmonic approximation}

In general, the crystal lattice periodicity implies a discrete translation symmetry such that a combined translation of electron and nuclei by any lattice vector does not change the many-body density of system. Therefore, any eigenfunction of the full many-body system, $\Psi_{\mathbf{k} n}(\mathbf{r}, \mathbf{R})$, can be characterized by a Bloch vector $\mathbf{k}$ in the first BZ

$$
\Psi_{\mathbf{k} n}(\mathbf{r}+\mathbf{a}, \mathbf{R}+\mathbf{a})=e^{i \mathbf{k} \cdot \mathbf{a}} \Psi_{\mathbf{k} n}(\mathbf{r}, \mathbf{R})
$$

The quasi-momentum transfers to any electronic wave function obtained by marginalizing the full wave function with an arbitrary nuclear wave function $\chi(\mathbf{R}+\mathbf{a})=\chi(\mathbf{R})$,

$$
\int d \mathbf{R} \chi^{*}(\mathbf{R}) \Psi_{\mathbf{k} n}(\mathbf{r}+\mathbf{a}, \mathbf{R})=e^{i \mathbf{k} \cdot \mathbf{a}} \int d \mathbf{R} \chi^{*}(\mathbf{R}) \Psi_{\mathbf{k} n}(\mathbf{r}, \mathbf{R}) .
$$

This allows us to extend the concept of the quasimomentum of electronic excitations and to establish a band structure useful for discussing the character of transition matrix elements for quantum crystals beyond the harmonic approximation.

\section{Born-Oppenheimer approximation}

Determining the quasi-momentum of electronic wave functions in the Born-Oppenheimer approximation based on the above considerations is not straightforward. Within the Born-Oppenheimer approximation, the electronic wave function, $\Phi_{\alpha}^{\mathbf{R}}(\mathbf{r})=\left\langle\mathbf{r} \mid \Phi_{\alpha}^{\mathbf{R}}\right\rangle$, is only determined up to an arbitrary phase $\varphi(\mathbf{R})$, which depends on the nuclear positions

$$
\Phi_{\alpha}^{\mathbf{R}}(\mathbf{r}) \simeq \Phi_{\alpha}(\mathbf{r} \mid \mathbf{R}) e^{i \varphi(\mathbf{R})} .
$$

Although $\varphi(\mathbf{R})$ can be fixed (up to a global constant) by requiring smooth changes with respect to adiabatic changes of the nuclear position, this is impractical for numerical computations.

Let us therefore consider that the Born-Oppenheimer wave function, Eq. (20), up to the unknown phase $\varphi(\mathbf{R})$ exactly coincides with the harmonic expansion, Eq. (15) and expand it into the components of the electronic eigenstates of the perfect crystal

$$
\begin{aligned}
\left\langle\Phi_{\mathbf{q} m}^{\mathbf{R}_{0}} \mid \Phi_{\alpha}^{\mathbf{R}}\right\rangle \simeq & {\left[\delta_{\mathbf{q k}} \delta_{m 0}+\left(1-\delta_{\mathbf{q k}} \delta_{m 0}\right) \frac{\left(\mathbf{R}-\mathbf{R}_{0}\right) \cdot \mathbf{A}_{\mathbf{k} 0}^{\mathbf{q} m}}{E_{\mathbf{k} 0}^{\mathbf{R}_{0}}-E_{\mathbf{q} m}^{\mathbf{R}_{0}}}\right] } \\
& \times e^{i \varphi(\mathbf{R})}
\end{aligned}
$$

The unknown phase $\varphi(\mathbf{R})$ can be eliminated considering the matrix elements

$$
\begin{array}{r}
\mathbf{T}\left(\mathbf{q}, m ; \mathbf{q}, m^{\prime}\right)= \\
\int d \mathbf{R}|\chi(\mathbf{R})|^{2}\left(\mathbf{R}-\mathbf{R}_{0}\right)\left\langle\Phi_{\alpha}^{\mathbf{R}} \mid \Phi_{\mathbf{q} m^{\prime}}^{\mathbf{R}_{0}}\right\rangle\left\langle\Phi_{\mathbf{q} m}^{\mathbf{R}_{0}} \mid \Phi_{\alpha}^{\mathbf{R}}\right\rangle \\
=\delta_{\mathbf{q k}}\left[\delta_{m^{\prime} 0}\left(1-\delta_{m 0}\right) \mathbf{t}_{\mathbf{k} 0}^{\mathbf{q} m}+\delta_{m 0}\left(1-\delta_{m^{\prime} 0}\right) \mathbf{t}_{\mathbf{k} 0}^{\mathbf{q} m^{\prime}}\right]
\end{array}
$$

for $m \neq m^{\prime}$ and $\mathbf{t}_{\mathbf{k} 0}^{\mathbf{q} m}$ denotes a vector matrix element which can be identified with the coefficients in the harmonic expansion on the r.h.s. of Eq.(21). Again, any 


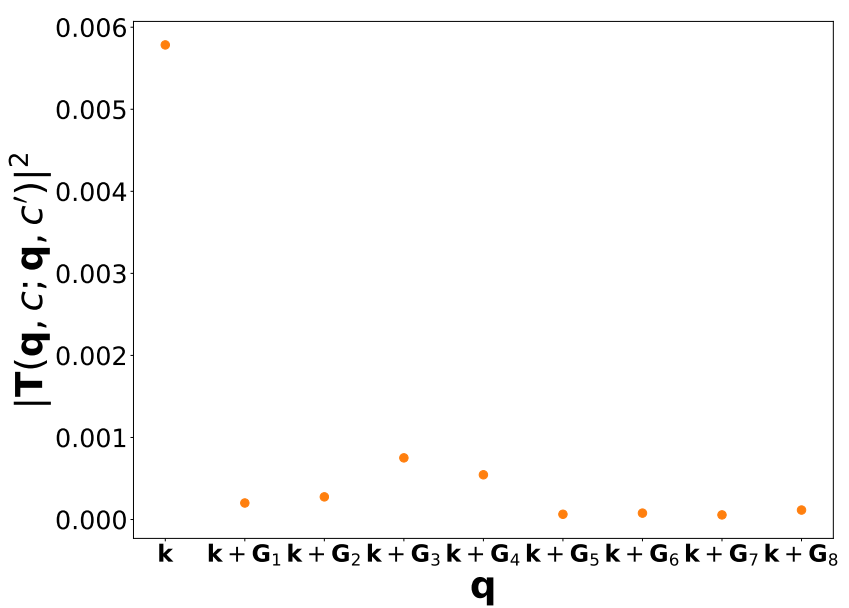

FIG. 2: Square modulus of the overlap (defined in eq. (22)) as a function of momentum $\mathbf{q}$ for a DFT-HSE calculation of hydrogen in the $\mathrm{C} 2 / \mathrm{c}-24$ structure at $T=200 \mathrm{~K}$ and $P=250 G P a . \mathbf{G}_{i}$ are the reciprocal lattice vectors of the supercell, $\mathbf{k}$ corresponds to the $\mathbf{X}+$ vector of the primitive BZ (see Fig. 3)

positive density distribution $\chi(\mathbf{R})^{2}$ with $\left\langle\mathbf{R}-\mathbf{R}_{0}\right\rangle=0$ for the nuclei can be used; in practice one simply averages over their equilibrium distribution at zero or finite temperature.

A non-vanishing $\left|\mathbf{T}\left(\mathbf{q}, m ; \mathbf{q}, m^{\prime}\right)\right|^{2}$ indicates that the Born-Oppenheimer electronic wave function, $\Phi_{\alpha}^{\mathbf{R}}$, averaged over the nuclear distribution transforms as an electronic wave function with crystal momentum q. Corrections beyond the harmonic approximation, as well as incomplete averaging over nuclear configurations may modify the results, but the matrix elements remain well peaked for a single crystal momentum, see Fig. 2 as an example.

For all the systems considered here, we have also verified that analysing $\mathbf{T}\left(\mathbf{q}, m ; \mathbf{q}, m^{\prime}\right)$ using DFT-HSE wave function gives the same quasi-momentum to the excitations as one would guess based on the corresponding ideal crystal states $\Phi_{\mathbf{k} n}^{\mathbf{R}_{0}}(\mathbf{r})$.

\section{Results: Hydrogen}

In this section we report the results of the electronic structure for solid hydrogen in the $\mathrm{C} 2 / \mathrm{c}-24$ structure at $200 \mathrm{~K}$ and $248 \mathrm{GPa}$. The ideal crystalline structure information has been obtained by $a b$ initio Random Structural Search method within the PBE approximation ${ }^{62}$ and further relaxed at constant pressure using the DFT-vdW-DF functional. We include zero point motion of the protons using path integrals with the CEIMC algorithm at constant volume and temperature. We consider a nearly cubic supercell of 96 protons with $L_{x}=11.12 a_{0}, L_{y}=$ $9.88 a_{0}, L_{z}=9.61 a_{0}$ where $a_{0}$ is the Bohr radius. Optimized Slater-Jastrow-backflow trial wave functions have been used for the CEIMC calculations ${ }^{49}$; details of the CEIMC simulations are reported in Ref. ${ }^{63}$. Averages over ionic positions for the electron addition and removal energies are obtained with 40 statistically independent configurations from the CEIMC trajectories.

For a given fixed nuclear configuration, the electron addition and removal energies are obtained by considering systems with a variable number of electrons $n=$ $N_{e}-N_{p} \in[-6,6]$. For each system we perform Reptation Quantum Monte Carlo (RQMC) calculations with imaginary-time projection $t=2.00 \mathrm{Ha}^{-1}$ and time step $\tau=0.01 \mathrm{Ha}^{-1}$ for a $6 \times 6 \times 6$ Monkhorst-Pack grid of twists. We checked that those values are adequate for converging the addition/removal energies within our resolution. The electron addition and removal energies are further corrected for finite size effects in leading and next-to-leading order $^{7}$.

Figure 3a illustrates the QMC highest occupied and lowest unoccupied bands at $200 \mathrm{~K}$ and for the perfect crystal plotted on top of the DFT/vdW-DF band structure of the unitary cell of $\mathrm{C} 2 / \mathrm{c}-24$ hydrogen crystal at $250 \mathrm{GPa}$. The DFT band structure was rigidly shifted ("scissor operator") to match the QMC gap. The QMC points are sparsely mapped onto the band structure of the primitive cell of crystalline hydrogen as we have only computed energies for a few twist values in order to determined the minimum insertion and removal energies for each twist value of our discrete grid of the supercell. Values for other momenta could be found by considering excitations at other twist values.

Figure 4 shows the GCTABC results for the electron volume density $n_{e}$ defined in Eq. (12) for solid hydrogen (with and without zero point motion). We also report the DFT-HSE integrated DOS computed with the procedure described in section II B. Vertical lines indicate the plateau region or, equivalently, the valence band maximum $\left(\mu^{-}\right)$ and conduction band minimum $\left(\mu^{+}\right)$of the quantum crystal reported in Fig. 3a.

The reduction of the gap due to nuclear motion is of the order of $2 \mathrm{eV}$; this big change is caused by its large zero point motion. More details on the electronic gaps of thermal hydrogen crystals can be found in Ref. ${ }^{19}$.

\section{OPTICAL PROPERTIES AT FINITE TEMPERATURE}

In the previous section, we have discussed how to determine the fundamental gap and the electronic band structure with explicitly correlated, many-body approaches, in particular with QMC methods. It is then natural to interpret the electron addition/removal energies as electron/hole excitations with well defined (electronic) quasi-momentum in the first BZ. The description of these excitations can be used in approximate single-particle theories, e.g. describing linear response spectral functions. In the following, we will focus on the calculation of optical properties computed within the Kubo-Greenwood (KG) formalism $^{64,65}$ taking into account the Born-Oppenheimer 


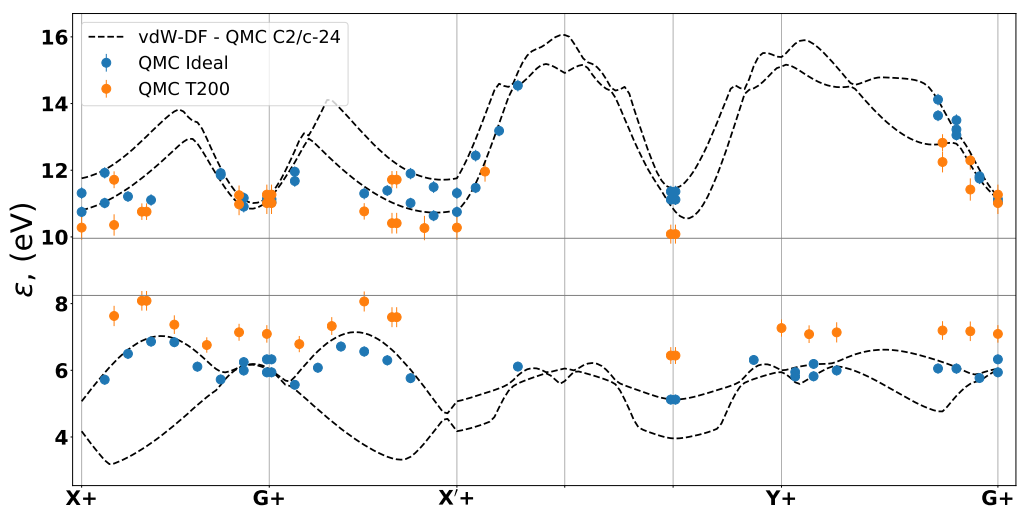

(a)

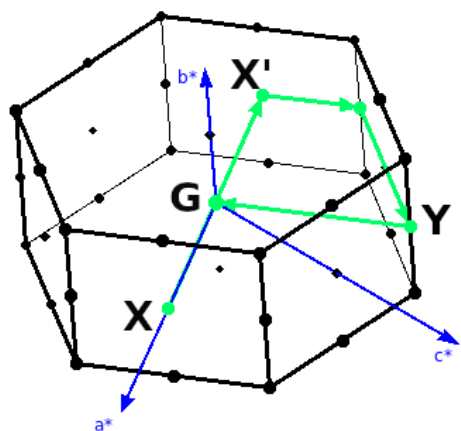

(b)

FIG. 3: (a) Band structure at finite temperature from QMC-CEIMC (orange points with $6 \times 6 \times 6$ twist grid) and for perfect crystal QMC (blue points with $8 \times 8 \times 8$ twist grid) calculations of 96 hydrogen atoms compared with the band structure from vdW-DF density functional for a unit cell of $\mathrm{C} 2 / \mathrm{c}-24$ hydrogen crystal at $248 \mathrm{GPa}$. The horizontal lines are the corresponding

valence band maximum $\left(\mu^{-}\right)$and conduction band minimum $\left(\mu^{+}\right)$of the thermal crystal determined in Fig. 4 . (b) The symmetry points of the Brillouin-Zone path. The actual path reported in panel (a) $\left(\mathbf{X}+, \mathbf{G}+, \mathbf{X}^{\prime}+, \mathbf{Y}+\right)$ was rigidly shifted from the one indicated in panel (b) by $\left(\frac{2 \pi}{16 L_{x}}, \frac{2 \pi}{16 L_{y}}, \frac{2 \pi}{16 L_{z}}\right)$ to better match the QMC twist grid.

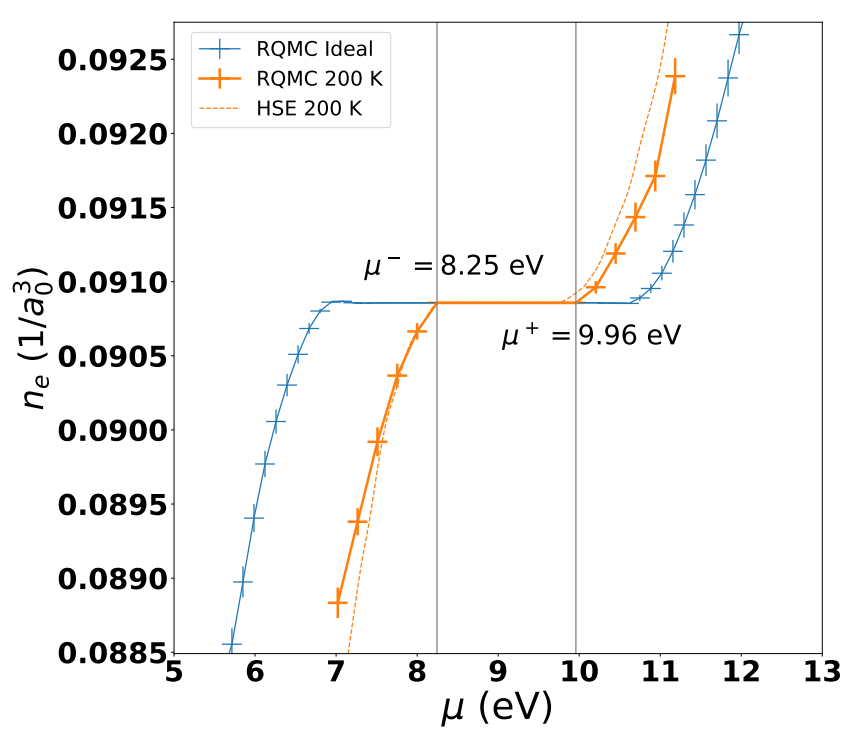

FIG. 4: Mean electron density from QMC-CEIMC calculations (orange solid line) and the integrated DOS computed with HSE density functional (orange dashed line) for $\mathrm{C} 2 / \mathrm{c}-24$ hydrogen crystal at $248 \mathrm{GPa}$ and $200 \mathrm{~K}$ plotted together with the RQMC electron density for a perfect hydrogen crystal (blue line).

dynamics of the nuclei.

\section{A. Semi-classical averaging}

Let us assume that the exact electron-nuclear wave function can be factorized into $|\alpha n\rangle \simeq\left|\Phi_{\alpha}^{\mathbf{R}}\right\rangle\left|\chi_{n}\right\rangle$, where
$\left\{\Phi_{\alpha}^{\mathbf{R}}, E_{\alpha}^{\mathbf{R}}\right\}$ is the (Born-Oppenheimer) solution of the electronic problem that depends parametrically on the nuclear configuration $\mathbf{R}$. Here $\left\{\left|\chi_{n}\right\rangle, \Omega_{n}\right\}$ are the nuclear wavefunctions and energies, and $\epsilon_{\alpha}^{\mathbf{R}}=E_{\alpha}^{\mathbf{R}}-E_{0}^{\mathbf{R}}$ denotes the Born-Oppenheimer excitation energy. Let us assume that the nuclear eigenstates are well described by the ground state Born-Oppenheimer energy surface $E_{0}(\mathbf{R})$, and neglect any the dependence on electronic excitations $\alpha \neq 0$.

Since the electrons can be considered to be in the ground state for the temperatures considered here, we can write the Kubo-Greenwood (KG) conductivity as a thermal average over nuclear states only,

$$
\sigma(\omega, T)=\frac{1}{Z} \sum_{n} e^{-\frac{\Omega_{n}}{k_{B} T}} \sigma_{n}(\omega),
$$

where $Z=\sum_{n} e^{-\Omega_{n} / k_{B} T}$. Within this theory, $\sigma_{n}(\omega)$ takes the form

$$
\begin{aligned}
\sigma_{n}(\omega) \propto & \frac{1}{\omega} \sum_{\alpha}^{\text {occ. unocc. }} \sum_{\beta, m}\left|\left\langle\chi_{n}\left|P_{\alpha \beta}^{\mathbf{R}}\right| \chi_{m}\right\rangle\right|^{2} \\
& \times \delta\left(\epsilon_{\beta m}-\epsilon_{\alpha n}-\hbar \omega\right)
\end{aligned}
$$

where $\alpha$ indicates the initial electronic states in the valence band $\left|\Phi_{\alpha}^{\mathbf{R}}\right\rangle, \beta$ and $m$ are the final electronic and nuclear states in the conduction band, respectively, $P_{\alpha \beta}^{\mathbf{R}}=\left\langle\Phi_{\alpha}^{\mathbf{R}}|\nabla| \Phi_{\beta}^{\mathbf{R}}\right\rangle$ the matrix element of the single electron momentum operator for a given (fixed) nuclear configuration $\mathbf{R}$, and $\epsilon_{\alpha n}=\left\langle\chi_{n}\left|\epsilon_{\alpha}^{\mathbf{R}}\right| \chi_{n}\right\rangle+\Omega_{n}$ is the total electron-nuclear excitation energies.

The conventional quasiclassical procedure introduced by Williams $^{51}$ and $\operatorname{Lax}^{52}$ (WL) substitutes the nuclear states $m$ with a continuum and replaces the eigenvalues $\epsilon_{\alpha n}$ in Eq. (24) by the electronic Born-Oppenheimer excitations 
for a fixed nuclear configuration $\epsilon_{\alpha}^{\mathbf{R}}$ (see refs. ${ }^{52,53}$ )

$$
\sigma_{n}^{W L}(\omega) \propto \frac{1}{\omega} \sum_{\alpha}^{\text {occ. unocc. }} \sum_{\beta}\left\langle\left.\chi_{n}|| P_{\alpha \beta}^{\mathbf{R}}\right|^{2} \delta\left(\Delta \epsilon_{\beta, \alpha}^{\mathbf{R}}-\hbar \omega\right) \mid \chi_{n}\right\rangle
$$

with $\Delta \epsilon_{\beta, \alpha}^{\mathbf{R}}=\epsilon_{\beta}^{\mathbf{R}}-\epsilon_{\alpha}^{\mathbf{R}}$. Using second order perturbation theory, it has been argued that this expression considers, in an effective way, the phonon-assisted indirect transitions ${ }^{53,54}$. However, for light nuclei such as hydrogen, replacing the nuclear spectrum by a classical continuum might not be accurate enough in the temperature regime explored by experiments.

\section{B. Quantum averaging}

An alternative procedure is to consider only direct transitions between pairs of electronic states of quantum or thermally averaged bands taking into account only nuclear zero point motion and thermal renormalizations of the bands, but neglecting phonon assisted transitions. In practice, we replace the eigenvalues in Eq.(24) by their quantum/thermal average neglecting explicit phonon contributions.

Considering low temperatures, nuclear states will occupy essentially only the nuclear ground state and a few low-lying phonon excitations, so that phononic energies can be neglected compared to typical electronic excitation energies

$$
\epsilon_{\beta m}-\epsilon_{\alpha n} \approx \epsilon_{\beta n}-\epsilon_{\alpha n}
$$

The summation over nuclear states $m$ can be replaced using the completeness relation $\sum_{m}\left|\chi_{\beta m}\right\rangle\left\langle\chi_{\beta m}\right|=1$,

$$
\begin{aligned}
\sigma_{n}(\omega) \propto & \frac{1}{\omega} \sum_{\alpha}^{\text {occ. unocc. }} \sum_{\beta}\left\langle\left.\chi_{\alpha n}|| P_{\alpha \beta}^{\mathbf{R}}\right|^{2} \mid \chi_{\alpha n}\right\rangle \\
& \times \delta\left(\epsilon_{\beta n}-\epsilon_{\alpha n}-\hbar \omega\right) .
\end{aligned}
$$

Within CEIMC the nuclear configurations, including zero point motion and phononic excitation at finite temperature, are sampled exactly according to the BornOppenheimer PES without any explicit phonon sampling, so that Eq. (27) cannot be applied directly. However, at zero temperatures, the nuclear average involved in calculating the matrix elements fully separates from the nuclear average in the electronic excitation energies. Assuming this factorization to hold also at low, but finite temperatures, we obtain

$$
\begin{aligned}
\sigma(\omega, T) \propto & \frac{1}{\omega} \sum_{\alpha}^{\text {occ. unocc. }} \sum_{\beta}\left\langle\left|P_{\alpha \beta}^{\mathbf{R}}\right|^{2}\right\rangle \\
& \times \delta\left(\left\langle\Delta \epsilon_{\alpha \beta}\right\rangle-\hbar \omega\right)
\end{aligned}
$$

We have obtained effectively a Kubo-Greenwood conductivity, where the $\delta$ function is represented as a gaussian with the eigenvalues and matrix elements averaged over the nuclear states. Eq. (28) is valid at low temperatures and when the transition matrix elements do not correlate with the eigenvalues. This effectively means that the transitions are computed for electronic eigenvalues averaged over the nuclear motion. We will call this procedure Quantum Averaging.

\section{Results: crystalline hydrogen and carbon diamond}

Here we illustrate the different renormalization procedures of the absorption spectra for solid C2/c-24 hydrogen at $200 \mathrm{~K}$ and for carbon diamond at $297 \mathrm{~K}$. While nuclear configurations of hydrogen are obtained as described in section IID for carbon diamond we used the cubic supercell containing 64 atoms with the lattice constant $3.56712 \AA$ appropriate for room temperature ${ }^{66}$. The nuclear configurations for carbon are obtained from the path integral molecular dynamics with the PBE functional using i-PI and QuantumEspresso ${ }^{67,68}$.

The absorption is computed for 40 fixed nuclear configuration within the Kubo-Greenwood formalism implemented in KGEC code ${ }^{69}$ using DFT. In hydrogen we used HSE functional with $8 \times 8 \times 8 \mathrm{k}$-grid and $2 \times 2 \times 2$ q-grid to sample the Fock operator, the kinetic energy cut-off was set to $40 \mathrm{Ry}$ and the gaussian smearing to $0.2 \mathrm{eV}$. In Carbon we used PBE functional with $10 \times 10 \times 10$ k-grid, 60 Ry of the cut-off and $0.35 \mathrm{eV}$ smearing. For both systems the PAW pseudopotentials were used.

Figure 5a shows the absorption spectra of hydrogen at 248 and $290 \mathrm{GPa}$ together with the absorption of ideal crystal at $250 \mathrm{GPa}$, computed with the HSE functional at the same conditions as for quantum crystals. The difference between the semiclassical and quantum averaging is particularly noticeable in the region of the onset of absorption (low energy/low absorption). The reduction of the gap at $248 \mathrm{GPa}$, given by the difference in the onset of the ideal and quantum absorption profiles is compatible with the reduction obtained by QMC in section II D.

The fundamental gap is often determined in experiments by using the Tauc extrapolation of absorption profiles $^{70}$. Figure 6 a illustrates the Tauc analysis of our absorption profiles for $\mathrm{C} 2 / \mathrm{c}-24$ hydrogen at $200 \mathrm{~K}$ at two pressures. The gap value is determined from the value of the intercept of a linear fit of $\sqrt{\omega \alpha}$ vs $\omega$ with the horizontal axis. At $290 \mathrm{GPa}$, the semi-classical procedure predicts the gap to be closed at variance with the fundamental gap of $\sim 0.4 \mathrm{eV}$ from the HSE band structure (orange vertical dashed line). The absorption profile by Quantum Averaging provides a more consistent value of the gap. At $248 \mathrm{GPa}$, the gap from the semi-classical absorption is $\sim 0.8 \mathrm{eV}$, about $0.5 \mathrm{eV}$ smaller than the fundamental gap from the HSE band structure (blue vertical dashed line). Again the gap extracted from the absorption profile by quantum averaging is in better agreement with the one from the band structure.

The results for carbon, which are shown in Fig. 5b, 


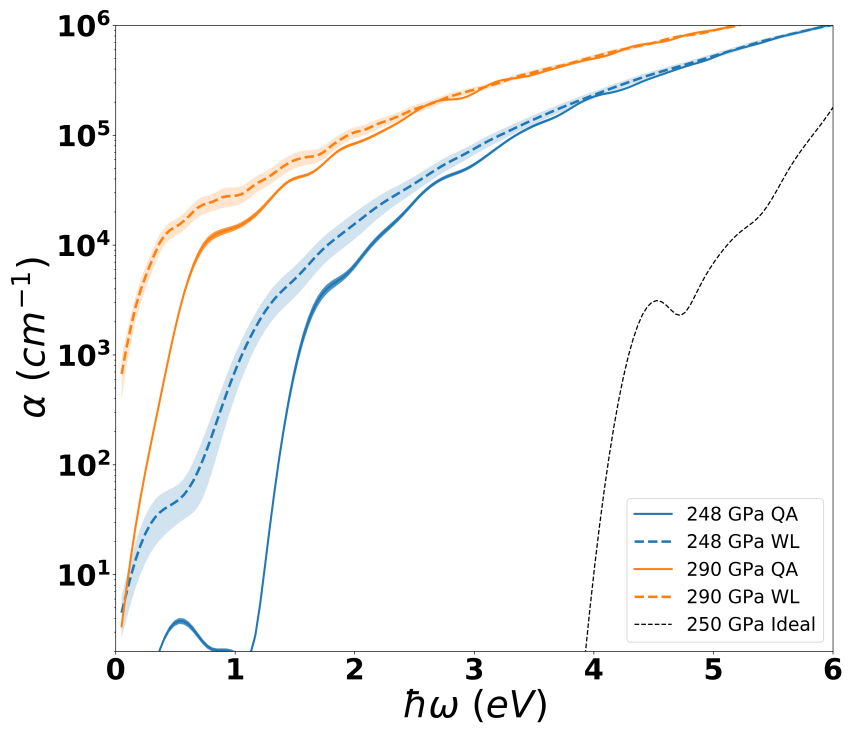

(a) Hydrogen

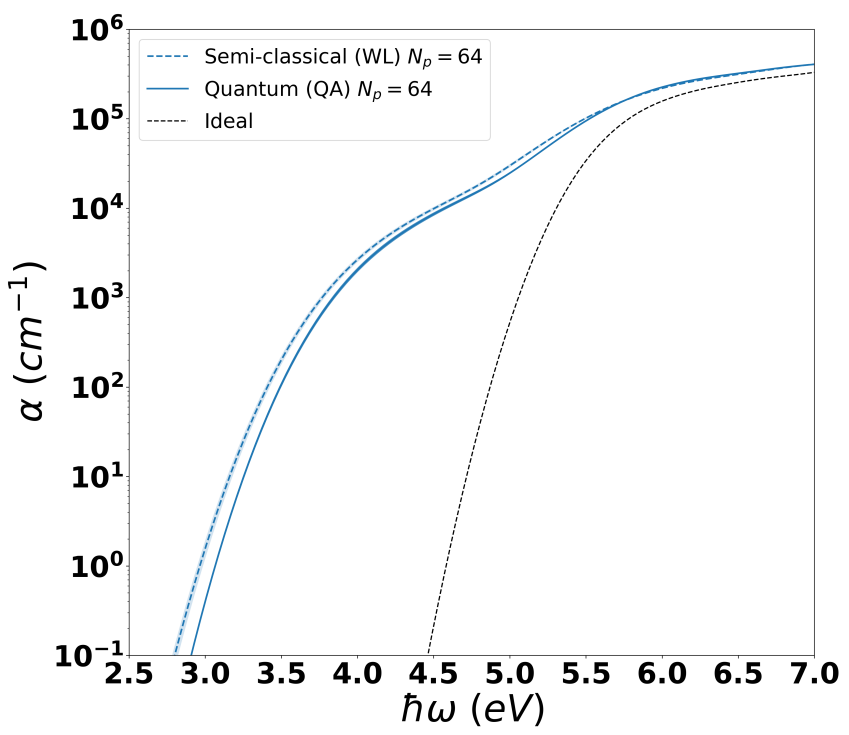

(b) Carbon

FIG. 5: Optical absorption for (a) C2/c-24 quantum crystals at 200K and (b) carbon diamond at $297 \mathrm{~K}$ using the semiclassical (WL) and quantum (QA) averaging procedures. The black dashed lines indicate the absorption of an ideal crystal.

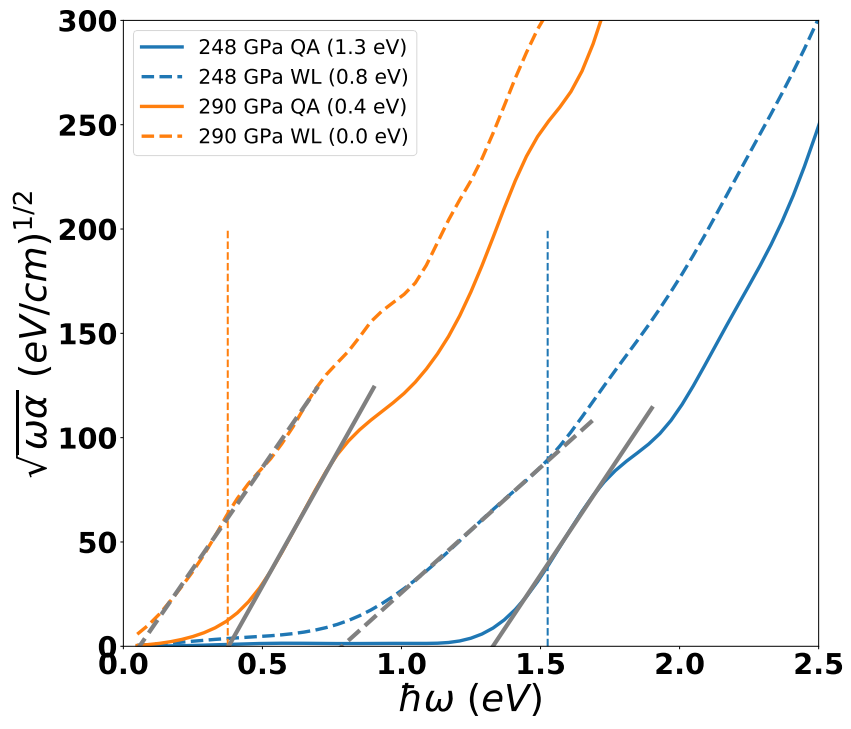

(a) Hydrogen

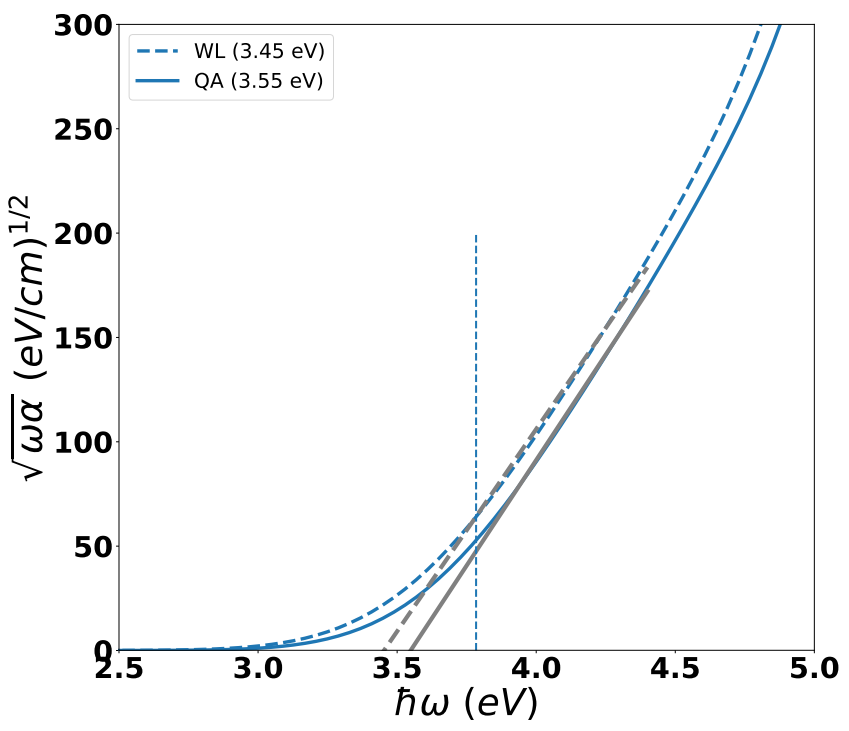

(b) Carbon

FIG. 6: Tauc analysis of the absorption profiles for (a) C2/c-24 quantum crystals at $200 \mathrm{~K}$ and (b) carbon diamond at $297 \mathrm{~K}$ using the semiclassical (WL) and quantum (QA) averaging procedures. Values of the gap extracted from the intercept of the linear fits and the horizontal axis are reported in the legend. The dashed vertical lines indicate the values of fundamental band-gaps computed using the HSE functional for hydrogen (a) and PBE for carbon (b).

demonstrate a smaller reduction from ideal to thermal crystal absorption. The results are compatible with the ones by Zachrias et al. Ref. ${ }^{54}$ up to a horizontal shift in the absorption profile used there to match the GW band gap. Since carbon is heavier than hydrogen, one expect the semiclassical averaging to become more accurate. From Fig. 5b we see that the difference between semiclassical and quantum averaging profiles is much smaller than in hydrogen. The gaps extracted from the absorption profiles for carbon, see Fig. 6b, are also compatible with the gap extracted from the PBE band structure. Note, however, that the gap values extracted from the Tauc analysis depend on the gaussian smearing in computing the absorption profile and on the fitting range used. 


\section{CONCLUSIONS}

In this work we have discussed electronic structure and optical properties for quantum crystals where quantum and thermal nuclear motion is important. We have focused on low temperatures assuming the validity of the Born-Oppenheimer approximation. First, we addressed the fundamental electronic gap in terms of electron addition and removal energies which can be directly computed using many-body Quantum Monte Carlo methods without relying on perturbation theory. We presented the methodology in the canonical and grand-canonical ensembles, the latter giving access to density of states. The averaging over motion of the ions is based on their thermodynamic definitions, e.g. the electronic (energy) density $n_{e}(\mu)(e(\mu))$. Therefore, the resulting energy gap might be different from a semi-classical approach where addition and removal energies for individual nuclear configurations are considered independently.

We then discussed how the electronic band-structure can be established for quantum crystals assigning to each addition/removal energy a well-defined crystal momentum for averages over nuclear equilibrium distributions. We presented an explicit procedure to determine the crystal momentum for electronic wave functions in the BornOppenheimer approximation without the need to explicitly fix the phase of the BO nuclear wavefunction.

We then discussed the calculation of optical conductivity using the Kubo-Greenwood formula. For light nuclei at low temperatures, we have shown that the semi-classical approximation should be replaced by a "quantum average" which takes into account the quantization of the nuclear motion, similar to the fundamental gap discussed before.

We have applied our methodology to study the band structure and density of states of hydrogen $\mathrm{C} 2 / \mathrm{c}-24$ crystal at $200 \mathrm{~K}$ and $250 \mathrm{GPa}$ where CEIMC has been used to sample the nuclear density matrix, and the QMC methods for the addition/removal energies.

Concerning optical properties, we have calculated the optical absorption for hydrogen and carbon diamond where we have approximated the electronic BornOppenheimer energies by DFT Kohn-Sham eigenvalues and discussed the differences between the quantum average and the semi-classical approximation. At low temperatures, only the quantum averaging procedure correctly reproduces the onset of absorption consistent with the one expected from the fundamental energy gap. As expected, in the case of carbon, the difference between the two methods are much smaller than for hydrogen.

This work paves the way for a more controlled and thermodynamically consistent investigation of electronic structure from many-body theory. Such procedures are particularly needed for strongly correlated systems which have important thermal and quantum ionic motions. The optical responses of such materials are one of the most basic and accurate experimental probes of their electronic properties. Hence simulation methods need to be able to consider all important effects to make unambiguous comparisons with experiment.

\section{ACKNOWLEDGMENTS}

This work has received funding from the European Union's Horizon 2020 research and innovation program under the grant agreement No. 676531 (project ECAM). D.M.C. was supported by DOE Grant NA DENA0001789 and by the Fondation NanoSciences (Grenoble). V.G. and C.P. were supported by the Agence Nationale de la Recherche (ANR) France, under the program "Accueil de Chercheurs de Haut Niveau 2015" project: HyLightExtreme. Computer time was provided by the PRACE Project 2016143296, ISCRAB (IsB17_MMCRHY,IscrB_FPSHPH) computer allocation at CINECA Italy, the high-performance computer resources from Grand Equipement National de Calcul Intensif (GENCI) Allocation 2018-A0030910282, and by the Froggy platform of CIMENT, Grenoble (Rhône-Alpes CPER07-13 CIRA and ANR-10-EQPX-29-01).

\section{DATA AVAILABILITY}

The data that support the findings of this study are available from the corresponding author upon reasonable request.

\section{Appendix A: Grand-canonical twist averaging boundary conditions}

Here we give the procedure to apply the grand-canonical twist averaging boundary conditions (GCTABC) ${ }^{56}$ for the total energy calculations with varying numbers of electrons and twist angles. At each twist angle, $\theta$, the electronic wave function obeys ${ }^{55}$

$$
\Psi\left(\mathbf{r}_{1}+L_{x} \hat{x} . ., \mathbf{r}_{N_{e}}\right)=e^{i \theta_{x}} \Psi\left(\mathbf{r}_{1}, . ., \mathbf{r}_{N_{e}}\right) .
$$

and is used to calculate the $N_{e}$ electron ground state energy $E_{0}\left(N_{e}, \theta\right)$.

At zero (electronic) temperature in the grand-canonical ensemble, the probability for a number, $N_{e}^{\theta}$ ) of electrons for a given twist angle $\theta$ is proportional to

$$
\begin{gathered}
\mathcal{P}\left(N_{e}^{\theta}\right) \quad \underset{\exp }{\longrightarrow}\left[-\beta\left(E_{0}\left(N_{e}, \theta\right)-\mu N_{e}\right)\right] \\
\underset{\beta \rightarrow \infty}{\longrightarrow} \min _{N_{e}}\left[E_{0}\left(N_{e}, \theta\right)-\mu N_{e}\right],
\end{gathered}
$$

where $\mu$ is the chemical potential.

In practice, within GCTABC, the ground state energy $E_{0}\left(N_{e}, \theta\right)$ is computed for different number of electrons, $N_{e}$, independently of the twist vector $\theta$. A uniform background charge is used to ensure global charge neutrality. Given $\mu$ and $\theta$ we can then determine $N_{e}^{\theta}$ from Eq. (A2). Equivalently, since $\partial E_{0}\left(N_{e}, \theta\right) / \partial N_{e}=\mu$ at the minimum of Eq. (A2), we can also scan the energy differences for 
each value of the chemical potential, $\mu$, and determine $N_{e}^{\theta}$ from bracketing

$E_{0}\left(N_{e}, \theta\right)-E_{0}\left(N_{e}-1, \theta\right) \leqslant \mu<E_{0}\left(N_{e}+1, \theta\right)-E_{0}\left(N_{e}, \theta\right)$,

resulting in an optimal number of electrons at each value of the chemical potential and twist $N_{e}^{\theta}(\mu)$.

To reduce finite size effects we further average over $M$ twist angles and divide by the volume of the supercell to obtain

$$
\begin{aligned}
e(\mu) & =\frac{1}{M V} \sum_{\theta} E_{0}\left(N_{e}^{\theta}, \theta\right) \\
n_{e}(\mu) & =\frac{1}{M V} \sum_{\theta} N_{e}^{\theta}(\mu)
\end{aligned}
$$

the electronic and energy density $n_{e}(\mu)$ and $e_{0}(\mu)$.

The resolution of the densities depends on the underlying twist grid, but the resolution of the chemical potential depends on the statistical error.

The twist grid error on the energy can be improved by calculating the Legendre transform of the grand potential as described in Ref. ${ }^{59}$ or may be fully eliminated by using symmetries and twist pockets as described in refs. ${ }^{56,58}$.

${ }^{1}$ S. Huotari, J. A. Soininen, T. Pylkkänen, K. Hämäläinen, A. Issolah, A. Titov, J. McMinis, J. Kim, K. Esler, D. M. Ceperley, M. Holzmann, and V. Olevano, "Momentum distribution and renormalization factor in sodium and the electron gas," Phys. Rev. Lett. 105, 086403 (2010).

${ }^{2}$ J. Kolorenč and L. Mitas, "Applications of quantum monte carlo methods in condensed systems," Reports on Progress in Physics 74, 026502 (2011).

${ }^{3}$ L. Shulenburger and T. R. Mattsson, "Quantum monte carlo applied to solids," Phys. Rev. B 88, 245117 (2013).

${ }^{4}$ L. K. Wagner and P. Abbamonte, "Effect of electron correlation on the electronic structure and spin-lattice coupling of high- $T_{c}$ cuprates: Quantum monte carlo calculations," Phys. Rev. B 90, 125129 (2014).

${ }^{5}$ L. K. Wagner and D. M. Ceperley, "Discovering correlated fermions using quantum monte carlo," Reports on Progress in Physics 79, 094501 (2016).

${ }^{6}$ R. J. Hunt, M. Szyniszewski, G. I. Prayogo, R. Maezono, and N. D. Drummond, "Quantum monte carlo calculations of energy gaps from first principles," Phys. Rev. B 98, 075122 (2018).

${ }^{7}$ Y. Yang, V. Gorelov, C. Pierleoni, D. M. Ceperley, and M. Holzmann, "Electronic band gaps from Quantum Monte Carlo methods," Physical Review B 101, 85115 (2020), arXiv:1910.07531.

${ }^{8}$ N. Hiraoka, Y. Yang, T. Hagiya, A. Niozu, K. Matsuda, S. Huotari, M. Holzmann, and D. M. Ceperley, "Direct observation of the momentum distribution and renormalization factor in lithium," Phys. Rev. B 101, 165124 (2020).

${ }^{9}$ Y. Yang, N. Hiraoka, K. Matsuda, M. Holzmann, and D. M. Ceperley, "Quantum monte carlo compton profiles of solid and liquid lithium," Phys. Rev. B 101, 165125 (2020).

${ }^{10}$ D. M. Ceperley and B. J. Alder, "Ground state of solid hydrogen at high pressures," Phys. Rev. B 36, 2092-2106 (1987).

${ }^{11}$ V. Natoli, R. M. Martin, and D. M. Ceperley, "Crystal structure of atomic hydrogen," Phys. Rev. Lett. 70, 1952-1955 (1993).

${ }^{12}$ M. Holzmann, C. Pierleoni, and D. M. Ceperley, "Coupled electron-ion monte carlo calculations of atomic hydrogen," Computer Physics Communications 169, $421-425$ (2005).

${ }^{13}$ C. Pierleoni, D. M. Ceperley, B. Bernu, and W. R. Magro, "Equation of state of the hydrogen plasma by path integral monte carlo simulation," Phys. Rev. Lett. 73, 2145-2149 (1994).
${ }^{14}$ W. Magro, D. Ceperley, C. Pierleoni, and B. Bernu, "Molecular dissociation in hot, dense hydrogen," Phys. Rev. Lett. 76, 12401243 (1996)

${ }^{15}$ B. Militzer and D. M. Ceperley, "Path integral monte carlo calculation of the deuterium hugoniot," Phys. Rev. Lett. 85, 1890-1893 (2000).

${ }^{16}$ C. Pierleoni, D. M. Ceperley, and M. Holzmann, "Coupled electron-ion monte carlo calculations of dense metallic hydrogen," Phys. Rev. Lett. 93, 146402 (2004).

${ }^{17}$ J. C. Grossman and L. Mitas, "Efficient quantum monte carlo energies for molecular dynamics simulations," Phys. Rev. Lett. 94, 056403 (2005).

${ }^{18} \mathrm{C}$. Attaccalite and S. Sorella, "Stable liquid hydrogen at high pressure by a novel ab initio molecular-dynamics calculation," Phys. Rev. Lett. 100, 114501 (2008).

${ }^{19}$ V. Gorelov, M. Holzmann, D. M. Ceperley, and C. Pierleoni, "Energy Gap Closure of Crystalline Molecular Hydrogen with Pressure," Physical Review Letters 124, 116401 (2020), arXiv:1911.06135.

${ }^{20}$ V. Gorelov, D. M. Ceperley, M. Holzmann, and C. Pierleoni, "Electronic energy gap closure and metal-insulator transition in dense liquid hydrogen," (2020), arXiv:2009.00652 [cond-mat.mtrlsci].

${ }^{21}$ F. Giustino, S. G. Louie, and M. L. Cohen, "Electron-phonon renormalization of the direct band gap of diamond," Phys. Rev. Lett. 105, 265501 (2010).

${ }^{22}$ A. Marini, "Ab initio finite-temperature excitons," Phys. Rev. Lett. 101, 106405 (2008).

${ }^{23}$ E. Cannuccia and A. Marini, "Effect of the quantum zero-point atomic motion on the optical and electronic properties of diamond and trans-polyacetylene," Phys. Rev. Lett. 107, 255501 (2011).

${ }^{24}$ E. Cannuccia and A. Marini, "Zero point motion effect on the electronic properties of diamond, trans-polyacetylene and polyethylene," The European Physical Journal B 85, 320 (2012).

${ }^{25}$ G. Antonius, S. Poncé, P. Boulanger, M. Côté, and X. Gonze, "Many-body effects on the zero-point renormalization of the band structure," Phys. Rev. Lett. 112, 215501 (2014).

${ }^{26} \mathrm{~S}$. Poncé, G. Antonius, Y. Gillet, P. Boulanger, J. Laflamme Janssen, A. Marini, M. Côté, and X. Gonze, "Temperature dependence of electronic eigenenergies in the adiabatic harmonic approximation," Phys. Rev. B 90, 214304 (2014).

${ }^{27}$ H. Kawai, K. Yamashita, E. Cannuccia, and A. Marini, "Electronelectron and electron-phonon correlation effects on the finitetemperature electronic and optical properties of zinc-blende gan," Phys. Rev. B 89, 085202 (2014).

${ }^{28}$ Poncé., Y. Gillet, J. Laflamme Janssen, A. Marini, M. Verstraete, and X. Gonze, "Temperature dependence of the electronic structure of semiconductors and insulators," The Journal of Chemical Physics 143, 102813 (2015), https://doi.org/10.1063/1.4927081.

${ }^{29}$ A. Molina-Sánchez, M. Palummo, A. Marini, and L. Wirtz, "Temperature-dependent excitonic effects in the optical properties of single-layer $\operatorname{mos}_{2}$," Phys. Rev. B 93, 155435 (2016).

${ }^{30}$ J. Menéndez, M. Noël, J. C. Zwinkels, and D. J. Lockwood, "Resonant indirect optical absorption in germanium," Phys. Rev. B 96, 121201 (2017).

${ }^{31}$ J. D. Querales-Flores, J. Cao, S. Fahy, and I. Savić, "Temperature effects on the electronic band structure of pbte from first principles," Phys. Rev. Materials 3, 055405 (2019).

${ }^{32}$ J.-M. Lihm and C.-H. Park, "Phonon-induced renormalization of electron wave functions," Phys. Rev. B 101, 121102 (2020).

${ }^{33} \mathrm{M}$. Zacharias and F. Giustino, "Theory of the special displacement method for electronic structure calculations at finite temperature," Phys. Rev. Research 2, 013357 (2020).

${ }^{34} \mathrm{P}$. B. Allen and V. Heine, "Theory of the temperature dependence of electronic band structures," Journal of Physics C: Solid State Physics 9, 2305-2312 (1976).

${ }^{35} \mathrm{P}$. B. Allen and M. Cardona, "Theory of the temperature dependence of the direct gap of germanium," Phys. Rev. B 23, 1495-1505 (1981). 
${ }^{36} \mathrm{P}$. B. Allen and M. Cardona, "Temperature dependence of the direct gap of si and ge," Phys. Rev. B 27, 4760-4769 (1983).

${ }^{37} \mathrm{~F}$. Giustino, "Electron-phonon interactions from first principles," Reviews of Modern Physics 89, 1-63 (2017), arXiv:1603.06965.

${ }^{38}$ M. A. Morales, J. M. McMahon, C. Pierleoni, and D. M. Ceperley, "Towards a predictive first-principles description of solid molecular hydrogen with density functional theory," Phys. Rev. B 87, 184107 (2013).

${ }^{39}$ N. D. Drummond, B. Monserrat, J. H. Lloyd-Williams, P. L. Ríos, C. J. Pickard, and R. J. Needs, "Quantum monte carlo study of the phase diagram of solid molecular hydrogen at extreme pressures," Nature Communications 6, 7794 (2015).

${ }^{40}$ B. Monserrat, E. A. Engel, and R. J. Needs, "Giant electronphonon interactions in molecular crystals and the importance of nonquadratic coupling," Phys. Rev. B 92, 140302 (2015).

${ }^{41}$ I. Errea, M. Calandra, and F. Mauri, "Anharmonic free energies and phonon dispersions from the stochastic self-consistent harmonic approximation: Application to platinum and palladium hydrides," Phys. Rev. B 89, 064302 (2014).

${ }^{42}$ L. Monacelli, I. Errea, M. Calandra, and F. Mauri, "Black metal hydrogen above $360 \mathrm{GPa}$ driven by proton quantum fluctuations," Nature Physics (2020), 10.1038/s41567-020-1009-3, arXiv:1912.05514.

${ }^{43}$ F. Della Sala, R. Rousseau, A. Görling, and D. Marx, "Quantum and thermal fluctuation effects on the photoabsorption spectra of clusters," Phys. Rev. Lett. 92, 183401 (2004).

${ }^{44}$ R. Ramírez, C. P. Herrero, and E. R. Hernández, "Path-integral molecular dynamics simulation of diamond," Phys. Rev. B 73, 245202 (2006).

${ }^{45}$ A. Franceschetti, "First-principles calculations of the temperature dependence of the band gap of si nanocrystals," Phys. Rev. B 76, 161301 (2007).

${ }^{46}$ R. Ramírez, C. P. Herrero, E. R. Hernández, and M. Cardona, "Path-integral molecular dynamics simulation of $3 c-$ SiC," Phys. Rev. B 77, 045210 (2008).

${ }^{47}$ G. Rillo, M. A. Morales, D. M. Ceperley, and C. Pierleoni, "Optical properties of high-pressure fluid hydrogen across molecular dissociation," Proceedings of the National Academy of Sciences of the United States of America 116, 9770-9774 (2019), arXiv: 1810.08131.

${ }^{48}$ M. A. Morales, C. Pierleoni, E. Schwegler, and D. M. Ceperley, "Evidence for a first-order liquid-liquid transition in high-pressure hydrogen from ab initio simulations," Proc. Nat. Acad. Sc. 107, 12799-12803 (2010).

${ }^{49}$ C. Pierleoni, M. a. Morales, G. Rillo, M. Holzmann, and D. M. Ceperley, "Liquid-liquid phase transition in hydrogen by coupled electron-ion Monte Carlo simulations," Proceedings of the National Academy of Sciences 113, 4954-4957 (2016).

${ }^{50}$ M. Zacharias, M. Scheffler, and C. Carbogno, "Fully anharmonic nonperturbative theory of vibronically renormalized electronic band structures," Physical Review B 102, 45126 (2020).

${ }^{51}$ F. E. Williams, "An absolute theory of solid-state luminescence," The Journal of Chemical Physics 19, 457-466 (1951).

${ }^{52} \mathrm{M}$. Lax, "The franck-condon principle and its application to crystals," The Journal of Chemical Physics 20, 1752-1760 (1952).

${ }^{53}$ C. E. Patrick and F. Giustino, "Unified theory of electronphonon renormalization and phonon-assisted optical absorption," Journal of Physics Condensed Matter 26 (2014), 10.1088/0953$8984 / 26 / 36 / 365503$.

${ }^{54}$ M. Zacharias and F. Giustino, "One-shot calculation of temperature-dependent optical spectra and phonon-induced bandgap renormalization," Phys. Rev. B 94, 075125 (2016).

${ }^{55}$ C. Lin, F. H. Zong, and D. M. Ceperley, "Twist-averaged boundary conditions in continuum quantum Monte Carlo algorithms," Physical Review E 64, 016702 (2001), arXiv:0101339 [cond-mat].
${ }^{56}$ S. Chiesa, D. M. Ceperley, R. M. Martin, and M. Holzmann, "Finite-size error in many-body simulations with long-range interactions," Physical Review Letters 97, 6-9 (2006).

${ }^{57}$ N. D. Drummond, R. J. Needs, A. Sorouri, and W. M. C. Foulkes, "Finite-size errors in continuum quantum monte carlo calculations," Phys. Rev. B 78, 125106 (2008).

${ }^{58}$ M. Holzmann, R. C. Clay, M. A. Morales, N. M. Tubman, D. M. Ceperley, and C. Pierleoni, "Theory of finite size effects for electronic quantum Monte Carlo calculations of liquids and solids," Physical Review B 94, 035126 (2016), arXiv:1603.03957.

${ }^{59}$ S. Azadi and W. M. C. Foulkes, "Efficient method for grandcanonical twist averaging in quantum monte carlo calculations," Phys. Rev. B 100, 245142 (2019).

${ }^{60}$ G. Rajagopal, R. J. Needs, A. James, S. D. Kenny, and W. M. C. Foulkes, "Variational and diffusion quantum monte carlo calculations at nonzero wave vectors: Theory and application to diamond-structure germanium," Phys. Rev. B 51, 10591-10600 (1995).

${ }^{61}$ M. D. Towler, R. Q. Hood, and R. J. Needs, "Minimum principles and level splitting in quantum monte carlo excitation energies: Application to diamond," Phys. Rev. B 62, 2330-2337 (2000).

${ }^{62}$ C. J. Pickard and R. J. Needs, "Structure of phase III of solid hydrogen," Nature Physics 3, 473-476 (2007).

${ }^{63}$ G. Rillo, M. A. Morales, D. M. Ceperley, and C. Pierleoni, "Coupled Electron-Ion Monte Carlo simulation of hydrogen molecular crystals," J. Chem Phys. 148, 102314 (2018).

${ }^{64} \mathrm{R}$. Kubo, "Statistical-mechanical theory of irreversible processes. i. general theory and simple applications to magnetic and conduction problems," Journal of the Physical Society of Japan 12, 570-586 (1957), https://doi.org/10.1143/JPSJ.12.570.

${ }^{65}$ D. A. Greenwood, "The Boltzmann Equation in the Theory of Electrical Conduction in Metals," Proc. Phys. Soc. 71, 585-596 (1958).

${ }^{66}$ S. Stoupin and Y. V. Shvyd'Ko, "Thermal expansion of diamond at low temperatures," Physical Review Letters 104, $2-5$ (2010).

${ }^{67}$ V. Kapil, M. Rossi, O. Marsalek, R. Petraglia, Y. Litman, T. Spura, B. Cheng, A. Cuzzocrea, R. H. Meißner, D. M. Wilkins, B. A. Helfrecht, P. Juda, S. P. Bienvenue, W. Fang, J. Kessler, I. Poltavsky, S. Vandenbrande, J. Wieme, C. Corminboeuf, T. D. Kühne, D. E. Manolopoulos, T. E. Markland, J. O. Richardson, A. Tkatchenko, G. A. Tribello, V. Van Speybroeck, and M. Ceriotti, "i-PI 2.0: A universal force engine for advanced molecular simulations," Computer Physics Communications 236, 214-223 (2019), arXiv:1808.03824.

${ }^{68}$ P. Giannozzi, O. Andreussi, T. Brumme, O. Bunau, M. B. Nardelli, M. Calandra, R. Car, C. Cavazzoni, D. Ceresoli, M. Cococcioni, N. Colonna, I. Carnimeo, A. D. Corso, S. de Gironcoli, P. Delugas, R. A. DiStasio, A. Ferretti, A. Floris, G. Fratesi, G. Fugallo, R. Gebauer, U. Gerstmann, F. Giustino, T. Gorni, J. Jia, M. Kawamura, H.-Y. Ko, A. Kokalj, E. KÃCEçÃCEkbenli, M. Lazzeri, M. Marsili, N. Marzari, F. Mauri, N. L. Nguyen, H.-V. Nguyen, A. O. de-la Roza, L. Paulatto, S. Poncé, D. Rocca, R. Sabatini, B. Santra, M. Schlipf, A. P. Seitsonen, A. Smogunov, I. Timrov, T. Thonhauser, P. Umari, N. Vast, X. Wu, and S. Baroni, "Advanced capabilities for materials modelling with quantum ESPRESSO," Journal of Physics: Condensed Matter 29, 465901 (2017).

${ }^{69}$ L. Calderín, V. V. Karasiev, and S. B. Trickey, "Kubo-Greenwood electrical conductivity formulation and implementation for projector augmented wave datasets," Comput. Phys. Commun. 221, 118-142 (2017), 1707.08437.

${ }^{70} \mathrm{~J}$. Tauc, R. Grigorovici, and A. Vancu, "Optical properties and electronic structure of amorphous germanium," Physica Status Solidi (b) 15, 627-637 (1966). 